\title{
Insulin Pump Failure is an Important Risk Factor for Diabetic Ketoacidosis in Pediatric Patients with Type 1 Diabetes
}

\author{
Junichi Suzuki*1 and Tatsuhiko Urakami ${ }^{2}$ \\ Department of Pediatrics, Nihon University School of Medicine, Japan
}

Received: May 01, 2018; Published: May 08, 2018

*Corresponding author: Tatsuhiko Urakami, Department of Pediatrics, Nihon University School of Medicine, 1-6 Kandasurugadai Chiyoda-ku, Tokyo 101-8309, Japan

Abbreviations: DKA: Diabetic Ketoacidosis; MDI: Multiple Daily Injections of Insulin

\section{Introduction}

Most of pediatric patients with type 1 diabetes are currently treated with intensive insulin therapy including multiple daily injections of insulin and insulin pump. Various reports have shown insulin pump is effective to improve glycemic control with decreasing hypoglycemia. However, insulin pump may be hazardous when pump problems occur, and the correspondence is wrong of problems. Even short interruption of insulin delivery can cause rapid rise in blood ketones accompanied by elevation of blood glucose. It is noteworthy that insulin pump failure can be an important risk factor for DKA during the management of diabetes in pediatric patients with type 1 diabetes.Diabetic ketoacidosis (DKA) is known to be a lethal condition, and the mortality rate of DKA in children and adolescents is reported to be $0.15-0.30 \%$ [1]. DKA can be found in both types 1 and type 2 diabetes and occurs not only at the onset but during the management. There is wide geographic variation in the frequency of DKA at the onset, and the frequencies range from approximately 15-70\% in Europe and North America [1]. The frequency of DKA during the management has been little reported, while risk factors for DKA on insulin treatment include failures in sick-day management, poor metabolic control, insulin omission, psychiatric (including eating) disorders, and insulin pump failures [1]. On the other hand, most of pediatric patients with type 1 diabetes are currently treated with intensive insulin therapy, such as multiple daily injections of insulin (MDI) and insulin pump. Use of insulin pump has been increasing worldwide particularly among children and adolescents, and various reports have shown the efficacy to improve glycemic control with decreasing hypoglycemia [2].

However, insulin pump therapy may be hazardous when pump problems occur, and the correspondence is wrong of problems. Even short interruption of insulin delivery can cause rapid rise in blood ketones accompanied by elevation of blood glucose. Interruption of basal insulin delivery for 30 minutes resulted in significant rise of blood glucose; i.e. $30 \mathrm{mg} / \mathrm{dL}$ (1.5 mmol/L) per 30 minutes [3]. It was also reported that pump stops for 5 hours in adult patients resulted in rise in beta-hydroxybutylate levels of 1-1.5 mmol/L. These results appear to be similar in children and adolescents [4]. Some studies have shown that risk of DKA on insulin pump was unchanged compared to when using MDI [5] or even lower in a recent long-term cohort study [6]. On the other hand, a literature review indicated an increased risk of DKA in pediatric patients using insulin pump in some studies [7]. We previously investigated the frequencies in DKA and their causes in the management of children and adolescents with type 1 diabetes during the period from 2003 to 2013 [8]. Frequencies of DKA in the study-period was $0.019 /$ patient/year, and DKA was more frequently found in patients using insulin pump than those treated with MDI. We found the most common cause of DKA was an insulin pump failure; mainly tube- and catheter-related failures, such as occlusion and winding, and accidental pull-out of catheters from insulin pump. Occlusion and winding of the tube and catheter resulted in the interruption of insulin delivery and DKA can develop within a few hours [9].

We recently again examined the causes and biochemical findings of DKA in children and adolescents with type 1 diabetes during the period from 2011 to 2017 (data not published). During the study-period, there were 18 episodes of DKA both at the onset and during the management. DKA occurred at the onset in 8 patients, when failures of sick-day management in 5 and when insulin pump failures in 5. The biochemical findings indicated no significant difference of the severity of ketoacidosis between at the onset and during the management (at the onset and during the management; 
HbA1c, 7.9, 8.3\%; blood glucose, 594, $597 \mathrm{mg} / \mathrm{dL}$; total ketones, 12,264.8, 9,170.0 $\mu \mathrm{mol} / \mathrm{L}$; venous blood pH 7.13, 7.20; HCO3-, 4.65, $11.30 \mathrm{mmol} / \mathrm{L}$ ). Half of DKA during the management was caused by insulin pump failures. From these results, it is concluded that insulin pump failure can be an important risk factor for occurrence of DKA during the management of diabetes in pediatric patients with type 1 diabetes. Frequent self-monitoring of blood glucose and measurement of ketones at home in a case with excessive hyperglycemia on insulin pump is necessary to prevent developing to DKA.

\section{Disclosure}

T.U. received honoraria from Novo Nordisk, Sanofi and Japan Chemical Research as a speaker and for attendance at advisory boards. JS declares no conflict of interest.

\section{References}

1. Wolfsdorf JI, Allgrove J, Craig ME, Edge J, Glaser N, et al. (2014) Diabetic ketoacidosis and hyperglycemic hyperosmolar state. Pediatr Diabetes 15 (20): 154-179.

2. Danne T, Bangstad HJ, Deeb L, JaroszChobot P, Mungaie L, et al. (2014) Insulin treatment in children and adolescents with diabetes. Pediatr Diabetes 15(20): 115-134.

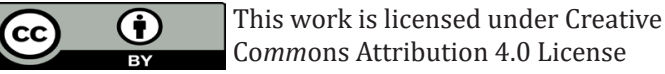

Submission Link: https://biomedres.us/submit-manuscript.php
3. Zisser $\mathrm{H}$ (2008) Quantifying the impact of a short-interval interruption of insulin-pump infusion sets on glycemic excursions. Diabetes Care 31(2): 238-239.

4. Hanas R, Lundqvist K, Windell L (2006) Blood glucose and betahydroxybutyrate responses when the insulin pump is stopped in children and adolescents. Pediatr Diabetes 7(5): 35.

5. Sulli N, Shashaj B (2006) Long-term benefits of continuous subcutaneous insulin infusion in children with type 1 diabetes: a 4-year follow-up. Diabet Med 23(8): 900-906.

6. Johnson SR, Cooper MN, Jones TW, Davis EA (2013) Long-term outcome of insulin pump therapy in children with type 1 diabetes assessed in a large population-based case-control study. Diabetologia 56(11): 23922400.

7. Hanas R, Ludvigsson J (2006) Hypoglycemia and ketoacidosis with insulin pump therapy in children and adolescents. Pediatr Diabetes $7(4): 32-38$

8. Urakami T, Habu M, Suzuki J (2014) DKA and severe hypoglycemia in management of type 1 diabetes during 2003-2013. Pediatr Int 56: 940.

9. Orsini-Federich M, Akwi JA, Canonico V, Celleno R, Ferolla P, et al. (2006) Early detection of insulin deprivation in continuous subcutaneous insulin infusion-treated patients with type 1 diabetes. Diabetes Technol Ther 8(1): 67-75.

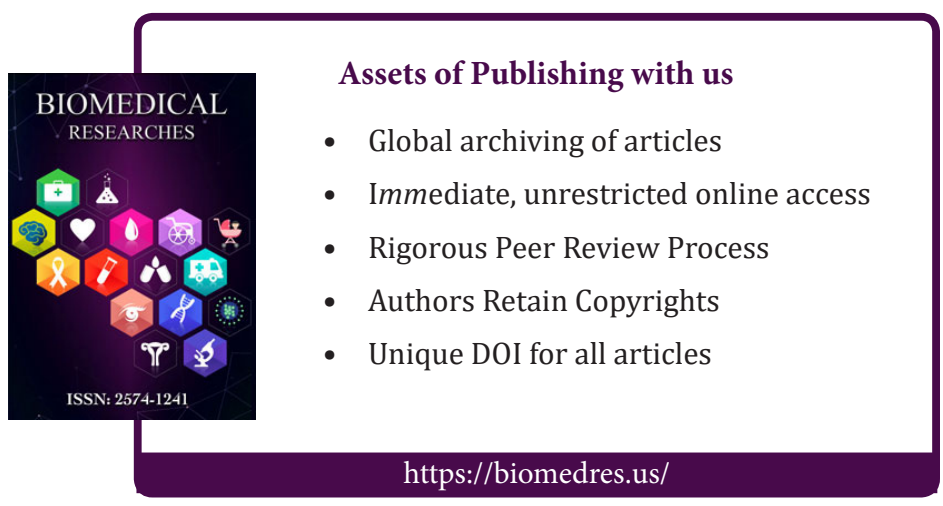

